\section{A Case of Disturbed Metabolism in Daphnia magna}

IN the first half of January this year, a marked decrease in the rate of reproduction was noticed in cultures of Daphnia magna that are being kept on a food of different algæ (mainly Scenedesmus sp.) at $18-20^{\circ} \mathrm{C}$. The algæe cultures are kept in large aquaria with fountain water with occasional addition of small quantities of nitrates and phosphates. These cultures are aerated artificially and illuminated with a normal light bulb.

Nothing else could be observed in the mother animals except the lack of active ovaria in many of them and a rather unusual amount of fat droplets. At the end of the same month the rate of reproduction had fallen to practically zero ; the mortality had also increased, and moulting took place only exceptionally. Closer observation of the animals revealed the fact that they were completely filled with fat droplets of a pink colour. These fat droplets formed a massive cover around the intestinal tract, from the first third of it down to the anus, while a great number of them could even be found at the base of the abdominal end claws. The thoracic appendixes were filled with droplets of the same colour, and they were even detected around the nephridia. Of the ovaries nothing could be seen. While well-fed Daphnia magna (with large fat reserves) can be starved for many days, during which they use up the fat, these abnormal animals would not consume their fat reserves during starvation, but died within about two days.

This irreversible fat-storage led to the idea that the Daphnia might be deficient in some substance, which either the algæ or the animals could not produce under the conditions offered to them. To find out whether there was really a kind of deficiency involved or not, the following experiments were made.

The animals were split into five groups of equal numbers: (1) and (2) the animals were kept as controls and continued to feed upon the mixture of algec as before; (3) besides algal food, the animals received finely dispersed egg-yolk for twelve hours every day; (4) instead of egg-yolk a suspension of baker's yeast was given for twelve hours every day; (5) the animals received the same food as before, but they were put twice a day under the light of a mercury arc (Original-Hanau Analysen-Quarzlampe) for five minutes at a time, at a distance of $20 \mathrm{~cm}$. from the arc, using a filter.

Results. After the first day of treatment the animals of groups (3) and (4) already showed increased vitality. After the second day of treatment first signs of resumed ovarian activity were observed in both groups. The control animals and those of group (5) showed no change in behaviour. After the third day all animals of group (3) had moulted and most of them carried summer-eggs. In group (4) most animals had moulted and showed active ovaria. The other animals continued to die and no moulting was observed. After the fourth day some animals of group (3) released young, and all animals were now carrying summer-eggs. Daphnia of group (4) showed eggs of the same type. Groups (1), (2) and (5) still showed no signs of improvement. After this, treatment was stopped and all animals were put back on normal food. While the animals of groups (1), (2) and (5) still showed the same pink colour as before, those of groups (3) and (4) looked pale; their fat reserves were of yellowish colour and reduced to normal size.
In the following days, some of the control animals and some of group (5) also showed some improvement-moulting and a slight egg-production.

Situation after three weeks. The animals of groups (3) and (4) have a high reproduction-rate (about 20 young per moult per animal); colour, yellowishbrown; fat storage small; about two-thirds of the animals have died since the beginning of the treatment. The control animals and those of group (5) have a poor reproduction (2-3 young per moult per animal); low vitality; pink colour, with abnormal large fat accumulations; of forty-five animals at the beginning of the experiment only three are still living.

It should be mentioned that during this period of abnormal behaviour the production of males or of sexual eggs was never noticed; this is true for the treated animals as well as for the untreated ones. The oxygen consumption, as measured by the Warburg technique, showed the following figures in c.mm. oxygen per 15 min. per mgm. dry weight : before and after metabolic disturbance, $M=2.97$ c.mm./ $15 \mathrm{~min} . / \mathrm{mgm}$., $\varepsilon= \pm 0.10$; during period of disturbed metabolism, $M=5.50$ c.mm. $/ 15 \mathrm{~min} . / \mathrm{mgm}$., $\varepsilon= \pm 0 \cdot 16$.

These results support the idea that the abnormal metabolism described above has its origin in some food deficiency. Of course, the complexity of the egg-yolk and of the yeast does not allow the type of deficiency to be specified.

Institute of Limnology,

Institute of Pharmacology, University of Lund. March 15.

\section{Heart-beat of the Hibernating Hedgehog}

THE most characteristic feature of the physiology of hibernation is the changing of a homoiothermic mammal within certain limits into a poikilothermic animal. Thus, for example, the body temperature of the hedgehog may decrease during hibernation to about $2^{\circ} \mathrm{C}$. When the animal wakes from hibernation, its body temperature rises in some hours from $2^{\circ}-5^{\circ} \mathrm{C}$. to $30^{\circ}-35^{\circ} \mathrm{C}$. again, and the animal returns to homoiothermy.

Owing to the low body temperature, the metabolism of the animal is greatly reduced during hibernation. This also has an effect on the heart function. The effect of hibernation on the physiology of the heart and the circulatory system has been but little investigated, however (cf., for example, Endres and collaborators $^{1}$, Ferdmann and Feinschmidt ${ }^{2}$, and Suomalainen and Sarajas ${ }^{3}$ ).

With the aid of electrocardiograms we found that the mean normal heart-beat of the hedgehog (bodyweight $420-920 \mathrm{gm}$.) is 188 per min. During hibernation (body-weight 420-770 gm., body temperature $6 \cdot 2-7 \cdot 7^{\circ} \mathrm{C}$.) the mean heart-beat was 21 per min. The reason for this is primarily the lowering of the body temperature and strengthening of vagus tone, probably also increase in magnesium, decrease in potassium, and increase in carbon dioxide content of the blood during hibernation.

\section{PaAvo SuOmalatNen}

SAMuli Sarajas

Zoological Laboratory,

University of Helsinki. April 5.

Endres, G., Matthews, B. H. C., Taylor, H., and Dale, Alison, Proc. Roy. Soc., B, 107, 222 (1931).

${ }^{2}$ Ferdmann, D., and Feinschmidt, O., Ergebn. Biol., 8, 1 (1932).

Suomalainen, Paavo, and Sarajas, Samuli, Ann. Zool. Soc. 'Vanamo', 14, 2,1 (1951). 